\title{
Enfermedad de
}

De Quervain. Anatomía

de la primera corredera

osteofibrosa y su influencia

en la etiopatogenia de

la enfermedad. Revisión

histórica y bibliográfica

\author{
J. M. ${ }^{\text {a Arandes Renú }}$ \\ MÉDICO CONSULTOR \\ SERVICIO COYT. UNIDAD DEL MIEMBRo SUPERIOR \\ HOSPITAL CLINIC. BARCELONA \\ Correspondencia: \\ Dr. José Ma Arandes \\ Hopital Clinic. Servicio COyT \\ C/ Villarroel, 170 \\ 08036 Barcelona \\ Teléfono 932275533 \\ e-mail: arandes@clinic.ub.es
}

Se revisa la descripción anatómica de la primera corredera osteofibrosa desde el punto de vista histórico, y la influencia que tiene el patrón anatómico sobre la etiopatogenia de la enfermedad de De Quervain. No parece existir una relación evidente entre el patrón anatómico y la enfermedad de De Quervain.

Palabras clave: anatomía primera corredera osteofibrosa; enfermedad de De Quervain; etiología.
Reviews the anatomical description of the first fibroosseous canal from the historical perspective, and the relationship to the anatomical patterns in the aetiology of de Quervain's disease.

No evidence appears to exist between the anatomical patterns and De Quervain's disease.

Key words: anatomy of the first fibroosseous canal; De Quervain's disease; etiology.

Rev. Iberam. Cir. Mano - Vol. 38 • Núm. 1 •Mayo 2010 (14-19)

E I n la enfermedad de De Quervain (EDQ), o estenosis de la primera corredera osteofibrosa (PCO), existe un aumento del grosor del retináculo, que se puede objetivar mediante estudio ecográfico o con resonancia magnética. Se considera que es más frecuente en los casos en los que existan tendones accesorios del abductor pollicis longus (APL) o del extensor pollicis brevis (EPB), o por la tabicación de la PCO. Otro factor etiológico es un vientre muscular del EPB excesivamente distal que penetre en la corredera. El propósito de este artículo es analizar el patrón anatómico de la PCO y su posible relación con la EDQ.

La primera descripción de la PCO, que hemos localizado en la revisión de los libros de anato- 


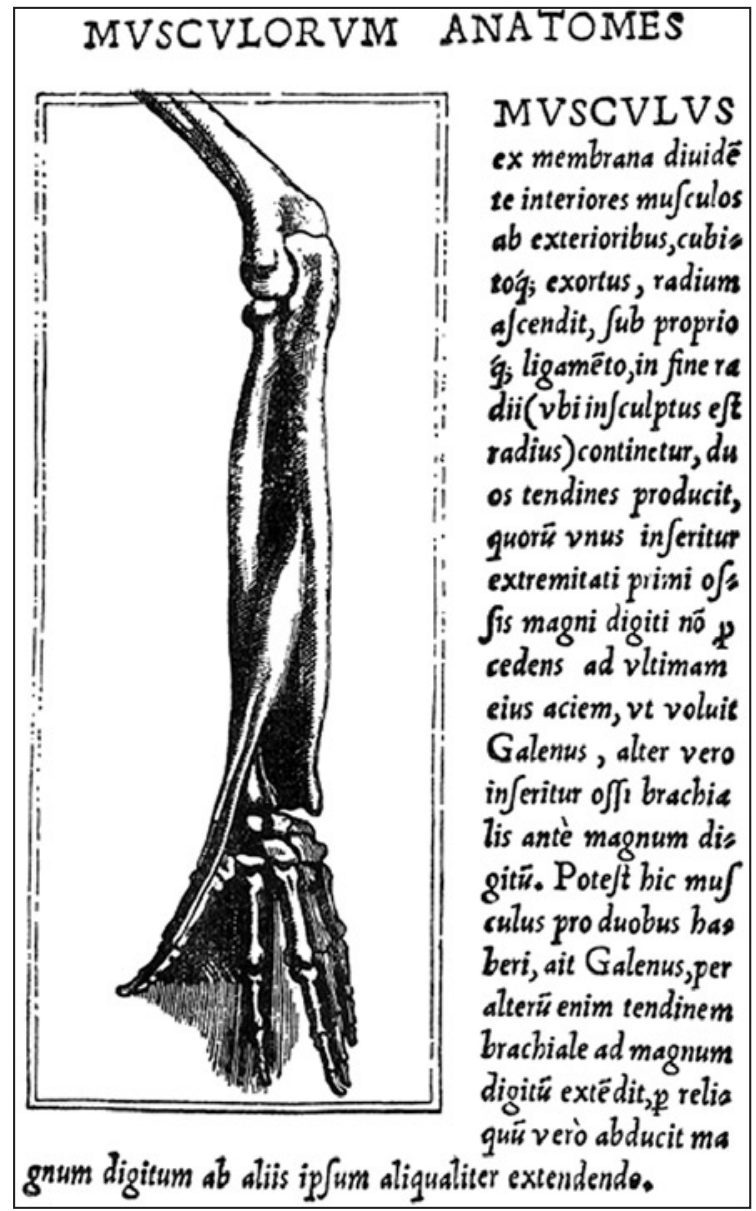

Figura 1: GB Canano. Humani Corporis Picturata Dissectio, 1541. Tabla 14. Descripción de la primera corredera.

mía «clásicos», es la de Giovanni Battista Canano ${ }^{1}$ (1515-1589), profesor de anatomía y médico del Papa Julio II, en su libro Musculorum Humani Corporis Picturata Dissectio publicado en Ferrara en el año 1541, que contiene 27 grabados de los músculos del brazo y antebrazo realizados por Girolano da Carpi (1501-1556). Quedan muy pocas copias de este libro (tres o cuatro) pero en 1925 se publicó en Firenze una edición facsímil ${ }^{2}$. En la tabla 14 dibuja el músculo extensor pollicis brevis y el abductor pollicis longus, y describe que pasan por un surco del radio en donde están mantenidos por un ligamento propio, y que la función de estos tendones es la de extender y abducir el dedo gordo (Figura 1):

Musculus ex membrana dividente interiores musculos ab exterioribus, cubitoque exortus, radium ascendit, sub propioque ligamento, in fine

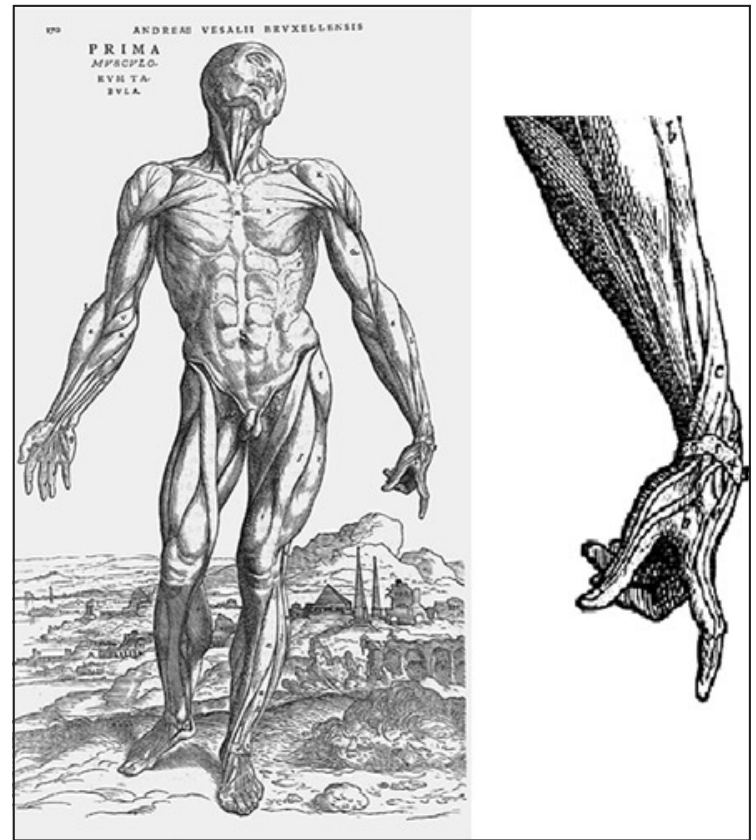

Figura 2: A Vesalio. De Humani Corporis Fabrica Libri VII. Pág 170. Numeración de las seis correderas de cubital a radial.

radii (ubi insculptus est radius) continetur, duos tendines producit, ...

Andrea Vesalio 3 (1514-1564) en su libro De Humani Corporis Fabrica Libri VII publicado en Basilea en 1543, dibuja en la página 170 el ligamento anular dorsal y las seis correderas osteofibrosas, numerándolas al revés de como las conocemos actualmente, pues las numera de cubital a radial (Figura 2).

Juan Valverde de Amusco (ca1525-ca1588) en su libro Historia de la Composición del Cuerpo Humano publicado en Roma en el año $1556^{4}$, editado en castellano, describe el ligamento anular dorsal denominándolo -en la página 46- atadura rezia y semejáte (por la parte de dentro) a un anillo, y dibuja y describe las correderas en la Tab segunda Libro segundo, numerándolas al igual que Vesalio de cubital a radial.

Alfred Velpeau en 1841 numera las correderas como las conocemos actualmente de radial a cubital.

Existen diversas variantes anatómicas de la PCO, describiéndose variantes tendinosas como la ausencia del EPB o la existencia de tendones accesorios del APL o del EPB, y variantes en cuanto a la tabicación de la $\mathrm{PCO}^{6}$. 


\section{VARIANTES TENDINOSAS EN LA PCO}

Albinus $^{7}$ en su libro Historia Musculorum Hominis Liber III publicado en 1734 fue el primero en emplear la denominación de abductor pollicis longus (abductore longo pollicis manus), y describe que el tendón del APL puede terminar en uno o dos tendones: Modo autem simplex manet, modo partitur se, \& non eadem in omnibus ratione. Y que cuando termina en dos tendones uno se inserta en el primer metacarpiano y el otro en la parte interna del trapecio.

Bonells y Lacaba ${ }^{8}$ en 1797 mencionan que el APL termina en dos tendones, pero que algunas veces se dividen en otros dos.

Sterling Bunnell en 1948 y en 1964, 10 observa la elevada frecuencia de tendones accesorios del APL en los casos intervenidos por EDQ. Encuentra en 12 de sus 22 casos la existencia de uno o más tendones accesorios del APL; un caso tenía cinco. En otro caso de la serie el tendón accesorio estaba enrollado por los otros tendones lo que ocasionaba un resalte.

Para Fenton ${ }^{11}$ el tendón accesorio del APL tiene siempre una disposición más volar.

Loomis ${ }^{12}$ describe cuatro variaciones anatómicas en los tendones de la PCO:

- el APL y el EPB tienen correderas separadas

- el APL y el EPB ocupan una única corredera

- un tendón accesorio pasa por una corredera más profunda, separada de la PCO para el APL y el EPB

- un tendón accesorio, generalmente del EPB, pasa por un canal que normalmente es más dorsal que la PCO. En estos casos el APL tiene un doble tendón.

Mellin et al. ${ }^{13}$, en un estudio anatómico, encontraron que la descripción clásica del APL sólo existe en el $20 \%$ de los casos. También Jackson et al. ${ }^{6}$ estudiando 300 muñecas de especímenes y 40 de pacientes intervenidos por enfermedad de De Quervain, concluyeron que en el 75\% de los especímenes la primera corredera es diferente a la considerada como estándar, y que la distribución de los tendones es simétrica en el $73 \%$ de los especímenes. Los resultados obtenidos en el estudio son:

- el EPB estaba ausente en seis especímenes, ninguno de los cuales tenían septo de separación. Cinco de éstos tenían dos tendones del APL. Esta forma no se encontró en ninguno de los pacientes intervenidos por EDQ.

- tenían un EPB 283 especímenes y 39 pacientes intervenidos.

- en los especímenes 77 tenían un APL, 171 dos, 28 tres, 6 cuatro y 1 cinco

- de los pacientes intervenidos: 13 uno, 13 dos, 12 tres, 1 cuatro y ninguno cinco

- encontraron dos EPB en:

- 11 especímenes: 5 con un APL, 5 con dos APL y 1 con tres

- uno sólo caso de los intervenidos tenía 2 EPB y 2 APL

- en dos casos un tendón accesorio del APL delgado se encontraba fuera de la PCO.

Sánchez Gómez et al. ${ }^{14}$ en una serie de 23 casos intervenidos por rizartrosis encontraron que el $95.7 \%$ de los pacientes tenían tendones accesorios del APL; 3 pacientes tenían tres tendones accesorios, 11 dos tendones accesorios y 8 pacientes un tendón accesorio; un paciente tenía un APL único aumentado de grosor.

\section{DIVISIÓN DE LA PRIMERA CORREDERA (Figura 3)}

Velpeau $^{5}$ en 1841 y Tillaux ${ }^{15}$ en 1880 llamaron la atención sobre la existencia de dos subcompartimentos separados para los dos tendones:

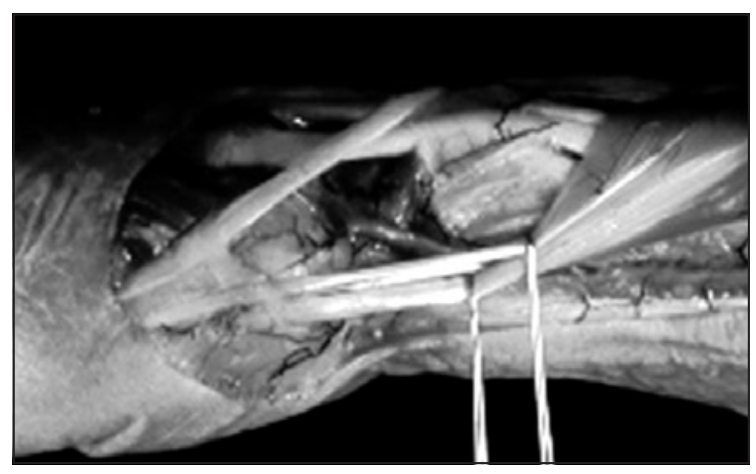

Figura 3: División de la PCO por un reborde óseo.

Revista Iberoamericana de Cirugía de la Mano 
Velpeau ${ }^{5}$ lo describe así:

En arrière du poignet, sous la peau et la couche souscutanée, on trouve une aponévrose qui forme une arcade rubanée très forte, destinée à brider les tendons auxquels elle fournit différentes gaînes; elle s'étend depuis l'apophyse stylö̈de du radius jusqu'à celle du cubitus: c'est le ligament annulaire postérieur du carpe; les canaux auxquels il donne naissance sont: $1^{\circ}$ en arrière et en dehors une coulisse très forte pour les tendons du court extenseur, du long abducteur du pouce, qui se dirigent en bas et en dehors; quand une cloison sépare ce canal en deux, c'est le tendon de l'extenseur qui se trouve en arrière;...

Tillaux ${ }^{15}$ lo describe de la siguiente manera:

Los tendones destinados al pulgar están dispuestos del modo siguiente: el abductor largo y el extensor corto forman... Se dirigen hacia abajo y adelante y afuera, pasan por detrás de la apófisis estiloides del radio, cruzan los dos músculos radiales, a los cuales cubren, y atraviesan una corredera fibrosa unas veces común, otras especial para cada uno, corredera esencialmente formada por un desdoblamiento del ligamento anular posterior del carpo...

Según la revisión de Jackson et al. ${ }^{6}$ el $40 \%$ de los casos tenían la primera corredera completa o parcialmente dividida por un septo. La presencia o ausencia de septo es simétrica en los dos lados en el 60\% de los especímenes. En los pacientes intervenidos el $67.5 \%$ tenían un septo de separación en la PCO, siendo la relación con la anatomía de los tendones:

- En los especímenes:

0 EPB en ninguno.

1 EPB: 25 un APL, 80 dos APL, 10 tres APL, 2 cuatro APL.

2 EPB: 1 un APL, 2 dos APL.

- En los pacientes intervenidos:

0 EPB ninguno.

1 EPB: 6 un APL, 9 dos APL, 10 tres APL, 1 cuatro APL.

2 EPB: 0 un APL, 1 dos APL.

En el artículo de Jackson et al. ${ }^{6}$ en dos casos un tendón accesorio del APL delgado se encontraba fuera de la PCO, ninguno de los dos tenía un septo de separación dentro de la PCO. Giles ${ }^{19}$ encontró este patrón en el 4\% de los casos intervenidos por EDQ. Loomis ${ }^{12}$ también describe este patrón, señalando que la corredera para este tendón accesorio del APL puede ser dorsal a la PCO o ser una subcorredera más profunda que la PCO.

En el estudio anatómico de Mellin et al. ${ }^{13}$ en la tercera parte de los especímenes la primera corredera está dividida por un septo, pudiendo existir dos ó más septos.

\section{RELACIÓN DEL PATRÓN ANATÓMICO DE LA PCO Y LA EDQ}

Loomis ${ }^{12}$ describe seis tipos posibles de estenosis en la PCO:

- Estenosis del APL y del EPB en una única PCO.

- Estenosis del APL y del EPB en la PCO, pero un tendón accesorio pasa por una corredera independiente más profunda.

- La estenosis afecta únicamente al tendón accesorio.

- el APL y el EPB pasan por correderas separadas, y la estenosis afecta a uno o a los dos tendones.

- La estenosis afecta al tendón accesorio que pasa por una corredera independiente que es más dorsal que la PCO.

- La estenosis afecta al tendón accesorio, que pasa por un canal independiente, y también afecta al APL y al EPB que pasan por la PCO que es común.

Burman ${ }^{16}$ describe la compresión localizada en un tendón accesorio del EPB que tenía un origen común.

Para Bahm et al. ${ }^{17}$ en el $76 \%$ de los casos intervenidos por EDQ existía más de un tendón del APL. Pero en el estudio anatómico y clínico de Jackson et al. ${ }^{6}$ no encuentran diferencias significativas entre el porcentaje de las variaciones tendinosas entre los pacientes intervenidos por EDQ y los especímenes, por lo que deducen que no es un factor que intervenga en la etiología de la EDQ.

La existencia de una separación en la PCO es más frecuente en los pacientes con EDQ que en la población general ${ }^{6,12}$ lo cual podría intervenir 


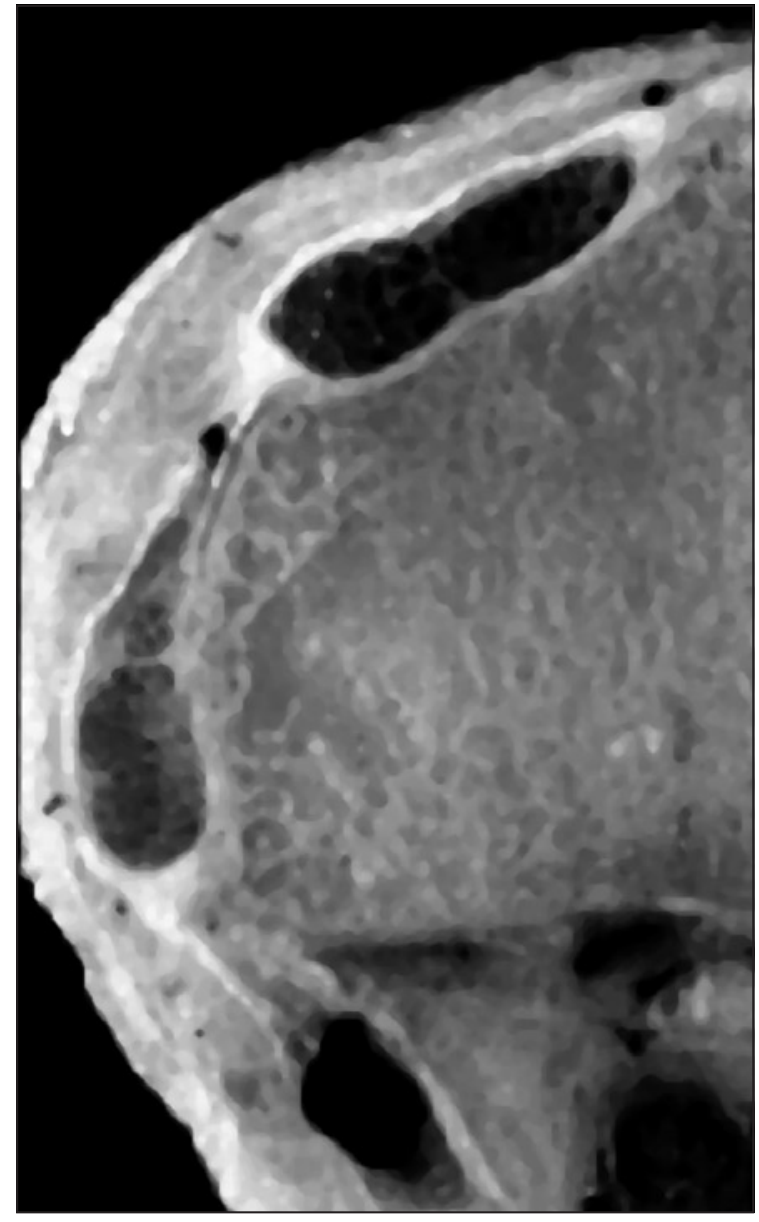

Figura 4: Primera y segunda correderas. Forma redondeada de la parte volar de la primera corredera osteofibrosa.

en la etiología de la EDQ. Sin embargo, un compartimento para el EPB y el APL independiente se encuentra ${ }^{18}$ en un $41 \%$ (rango $\left.=20-75\right)$ de las disecciones anatómicas en seis estudios ${ }^{6,19-23}$ y en un $47 \%$ (rango $=25-68$ ) de las intervenciones quirúrgicas en cuatro estudios $6,21,24,25$.

Para Bahm et al. ${ }^{17}$ en un $60 \%$ de casos intervenidos de EDQ la primera corredera era doble, pero es una incidencia similar a la encontrada por Sánchez Gómez et al. ${ }^{14}$ en una serie de 23 casos intervenidos por rizartrosis en los que encontraron un compartimento independiente para el EPB en el $54 \%$ de los casos.

La existencia de un PCO tabicado, para algunos, podría ser la causa del fallo del tratamiento mediante infiltraciones ${ }^{18,26}$.

Yuasa y Kiyoshige ${ }^{27}$ basándose en que la EDQ está originada únicamente por la compresión de la subcorredera del EPB - hecho ya descrito por Shepherd ${ }^{28}$ y por Murphy ${ }^{29}$ - en una serie de 22 pacientes intervenidos, a los 16 que tenían un septo de separación entre los dos tendones les seccionaban únicamente la subcorredera del EPB, consiguiendo en todos los casos la desaparición de la sintomatología.

Kauer y Landsmeer ${ }^{30}$ analizan la forma de las correderas osteofibrosas del radio encontrando que tienen formas diferentes, pero la porción de las correderas que tiene función de polea son redondeadas. En PCO la parte redondeada es la más volar, por lo que la función de polea únicamente la realiza la PCO para el EPB, porque la parte de la PCO para el APL no es redondeada (Figura 4). Esto explicaría que la patología se encuentre en el EPB y no en el APL, e iría a favor de la teoría traumática; pero en contra está que el engrosamiento de la PCO en la EDQ es uniforme en toda la corredera, no exclusivamente de la parte que corresponde al EPB, lo que apoyaría la teoría no traumática.

Los estudios anatómicos demuestran que la primera corredera tiene una disposición anatómica simétrica en el $73 \%$ de los $\operatorname{casos}^{6}$, pero no hay diferencias en la frecuencia de EDQ entre la mano dominante y no dominante ${ }^{24,31-34}$, y la EDQ rara vez es bilateral; Keppler ${ }^{35}$ presenta dos casos y Winterstein ${ }^{36}$ cuatro.

Posiblemente también puedan influir otros patrones anatómicos como la longitud de la PCO o su inclinación con respecto al eje de los tendones del APL o del EPB, pero un elevado porcentaje de EDQ, alrededor del $70 \%$, curan con una o dos infiltraciones de corticoides sin recidivas posteriores, y el patrón anatómico sigue siendo el mismo.

La EDQ tiene una mayor incidencia en mujeres, pero la anatomía y las solicitaciones que sufre la PCO es semejante en ambos sexos, por lo que en la etiopatogenia puede intervenir un factor hormonal/metabólico.

Por otra parte, la EDQ es muy frecuente en las embarazadas, en el periodo del pre- o postparto, y se puede observar en pacientes en tratamiento con fluorquinolonas, y en ninguno de los dos casos influye la anatomía de la PCO.

Desde el punto de vista anatómico y clínico no existe una evidencia en cuanto a la influencia del patrón de la PCO en la etiopatogenia de la EDQ. 


\section{BIBLIOGRAFÍA}

1. Canano IB. Musculorum Humani Corporis Picturata Dissectio. Ferrara. 1543: Tabla XIV.

2. Canano IB. Musculorum Humani Corporis Picturata Dissectio. Facsimile Ed. annotated by $\mathrm{H}$ Cushing \& EC Streeter. Florence: R Lier \& Co, 1925: Tabla XIV.

3. Vesalius A. De Humani Corporis Fabrica Libri VII. Lib II. Bâle: Ioannum Oporinum, 1573: 170.

4. Valverde de Amusco J. Historia de la Composición del Cuerpo Humano. Roma: Antonio de Salamanca, 1556: 46; Tab 2 Libro 2.

5. Velpeau AFNFP. Crépitation douloureuse des tendon. En: Leçons Orales de Clinique Chirurgicale. Recuellies et Publiées par M le Docteur P. Pavillon. t I. Paris: Germer Baillière, 1841: 94-111.

6. Jackson WT, Viegas SF, Coon $\mathrm{TM}$, et al. Anatomical variations in the first extensor compartment of the wrist. $\mathrm{J}$ Bone Joint Surg Am, 1986; 68: 923-6.

7. Albinus BS. Historia MuscuIorum Hominis. Liber III, Cap CLIX, Leidae Batavorum: Theodorum Haak \& Henricum Mulhovium, 1734: 460-3.

8. Bonells J, Lacaba I. Curso completo de Anatomía del Cuerpo Humano. T II. Madrid: En la Imprenta de Sancha, 1797: 96.

9. Bunnell S. Surgery of the Hand. Second Ed. Philadelphia: JB Lippincott Co, 1948: 567-73.

10. Bunnell S. Surgery of the Hand. Fourth Ed. Philadelphia: JB Lippincott Co, 1964: 472-3.

11. Fenton R. Stenosing tendovaginitis at the radial styloid involving an accesory tendon sheath. Bull Hosp Joint Dis, 1950; 11: 90-5.

12. Loomis LK. Variations of stenosing tenosynovitis at the radial styloid process. J Bone Joint Surg Am, 1951; 33: 340-6.

13. Melling M, Wilde J, Schnallinger $M$, et al. Supernumerary tendons of the abductor pollicis. Acta Anatomica, 1996; 155: 291-4.

14. Sánchez Gómez P, Fuentes Días A, Lajara Marco F, et al. Resultados del tratamiento de la rizartrosis mediante sección de los tendones accesorios del abductor pollicis longus. Rev Iberamer Cir Mano, 2007; 35: 27-38.

15. Tillaux P. Tratado de Anatomía Topográfica Aplicada a la Cirugía. Barcelona: Editorial Espasa Hermanos, 1880: 586-8.

16. Burman M. The separate stenosis of an anomalous extensor tendon of the thumb in de Quervain's disease. New York J Med, 1951; 51: 2417.

17. Bahm J, Szabo Z, Foucher G. The anatomy of the Quervain's disease. A study of operative findings. Int Orthop (SICOT), 1995; 19: 209-11.

18. Zingas C, Failla JM, van Holsbeeck M. Injection accuracy and clinical relief of de Quervain's tendinitis. J Hand Surg Am, 1998; 23: 89-96.

19. Giles KW. Anatomical variations affecting the surgery of the De Quervain's disease. J Bone Joint Surg Br, 1960; 42: 352-5.

20. Gonzalez $\mathrm{MH}$, Sohlberg $\mathrm{R}$, Brown A, et al. The first dorsal extensor compartment: an anatomic study. J Hand Surg Am, 1995; 20: 657-60.

21. Minamikawa Y, Peimer CA, Cox WL, et al. De Quervain's syndrome and anatomical studies of the fibroosseous canal. Orthopedics, 1991; 14: 545-9.

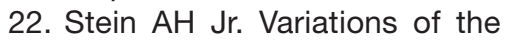
tendons of insertions of the abductor pollicis longus and extensor pollicis brevis. Anat Rec, 1951; 110: 49-55.

23. Leão L. De Quervain's disease. A clinical and anatomical study. J Bone Joint Surg Am, 1958; 40: 1063-70.

24. Finkelstein H. Stenosing tendovaginitis at radial styloid process. J Bone Joint Surg, 1930; 12: $509-40$.
25. Strandell G. Variations of the anatomy in stenosing tenosynovitis at radial styloid process. Acta Chir Scand, 1957; 113: 234-40.

26. Louis DS. Incomplete release of the first dorsal compartment - a dignostic test. J Hand Surg Am, 1985; 12: 87-8.

27. Yuasa K, Kiyoshige Y. Limited surgical treatment of the Quervain's disease: Decompression of only the extensor pollicis brevis subcompartment. J Hand Surg Am, 1998; 23: 840-3.

28. Shepherd JA. Constriction of the extensor pollicis brevis tendon. An unusual lesion simulating de Quervain's disease. Br J Surg, 1946; 34: 213-4.

29. Murphy ID. An unusual form of de Quervain's syndrome; report of two cases. J Bone Joint Surg Am, 1949; 31: 858-9.

30. Kauer JMG, Landsmeer JMF. Le poignet. En: Tubiana $R(E d)$. Traité de Chirurgie de la Main. Vol. 1. Paris: Masson, 1980: 176-91.

31. Lipscomb PR. Stenosing tenosynovitis at the radial styloid process (de Quervain's disease). Ann Surgery, 1951; 134: 110-5.

32. Lamphier TA, Pepi JF, Brush $\mathrm{CH}$, et al. De Quervain's disease. J Int Coll Surg, 1959; 31: 192.

33. Faithfull DK, Lamb DW. De Quervain's disease. A clinical review. The Hand, 1971; 3: 2331.

34. Kay NRM. De Quervain's disease. Changing pathology or changing perception?. J Hand Surg Br, 2000; 25: 65-9.

35. Keppler W. Zur Klinik des stenosierenden Tendovaginitis am Processus styloideus radii. Med Klin, 1917; 13: 1014.

36. Winterstein O. Zur Tendovaginitis stenosans am Processus styloïdeus radii (de Quervain). Münch Med Wochensch, 1927; 64: 12-5. 